\title{
Characteristic mTOR activity in Hodgkin-lymphomas offers a potential therapeutic target in high risk disease - a combined tissue microarray, in vitro and in vivo study
}

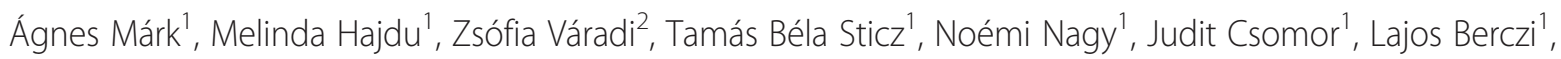
Viktória Varga ${ }^{1}$, Monika Csóka², László Kopper ${ }^{1}$ and Anna Sebestyén ${ }^{1,3^{*}}$

\begin{abstract}
Background: Targeting signaling pathways is an attractive approach in many malignancies. The PI3K/Akt/mTOR pathway is activated in a number of human neoplasms, accompanied by lower overall and/or disease free survival. mTOR kinase inhibitors have been introduced in the therapy of renal cell carcinoma and mantle cell lymphoma, and several trials are currently underway. However, the pathological characterization of mTOR activity in lymphomas is still incomplete.

Methods: mTOR activity and the elements of mTOR complexes were investigated by immunohistochemistry on tissue microarrays representing different human non-Hodgkin-lymphomas (81 cases) and Hodgkin-lymphomas (87 cases). The expression of phospho-mTOR, phospho-4EBP1, phospho-p70S6K, phospho-S6, Rictor, Raptor and Bcl-2, Bcl-xL, Survivin and NF-kappaB-p50 were evaluated, and mTOR activity was statistically analyzed along with 5-year survival data. The in vitro and in vivo effect of the mTOR inhibitor rapamycin was also examined in human Hodgkin-lymphoma cell lines.

Results: The majority (>50\%) of mantle cell lymphoma, Burkitt lymphoma, diffuse large B-cell lymphoma, anaplastic large-cell lymphoma and Hodgkin-lymphoma cases showed higher mTOR activity compared to normal lymphoid tissues. Hodgkin-lymphoma was characterized by high mTOR activity in $93 \%$ of the cases, and Bcl-xL and NF-kappaB expression correlated with this mTOR activity. High mTOR activity was observed in the case of both favorable and unfavorable clinical response. Low mTOR activity was accompanied by complete remission and at least 5-year disease free survival in Hodgkin-lymphoma patients. However, statistical analysis did not identify correlation beetween mTOR activity and different clinical data of HL patients, such as survival. We also found that Rictor (mTORC2) was not overexpressed in Hodgkin-lymphoma biopsies and cell lines. Rapamycin inhibited proliferation and induced apoptosis in Hodgkin-lymphoma cells both in vitro and in vivo, moreover, it increased the apoptotic effect of chemotherapeutic agents.

(Continued on next page)
\end{abstract}

\footnotetext{
* Correspondence: anna@korb1.sote.hu

${ }^{1} 1$ st Department of Pathology and Experimental Cancer Research,

Semmelweis University, Üllői út 26, Budapest 1085, Hungary

${ }^{3}$ Tumor Progression Research Group of Joint Research Organization of the

Hungarian Academy of Sciences and Semmelweis University, Budapest,

Hungary

Full list of author information is available at the end of the article
} 
(Continued from previous page)

Conclusions: Targeting mTOR activity may be a potential therapeutic tool in lymphomas. The presence of mTOR activity probably indicates that the inclusion of mTOR inhibition in the therapy of Hodgkin-lymphomas may be feasible and beneficial, especially when standard protocols are ineffective, and it may also allow dose reduction in order to decrease late treatment toxicity. Most likely, the combination of mTOR inhibitors with other agents will offer the highest efficiency for achieving the best clinical response.

Keywords: mTOR activity, Hodgkin-lymphoma, Rapalogs, TMA, Hodgkin-lymphoma xenograft

\section{Background}

The number of patients diagnosed with lymphoid malignancies has increased to 18,000 per year in Europe [1]. Hodgkin-lymphomas (HL) with characteristic histopathological subtypes comprise about $11 \%$ of all lymphomas $[1,2]$. Tumor cells [Hodgkin-/Reed-Sternberg (HRS) cells] usually represent only a small fraction of diagnostic histology, while differences in microenvironment (reactive lymphocytes, extracellular matrix) allow subclassification of $\mathrm{HL}[3,4]$. The prognosis of $\mathrm{HL}$ patients is relatively good, however, some patients may relapse in spite of first line chemotherapy and radiation protocols, and can be further treated, sometimes cured by intensified chemotherapy and/or peripheral stem cell transplantation [5]. Unfortunately, these treatments still fail in $15-20 \%$ of $\mathrm{HL}$ patients [6]. Considering that the majority of HL patients are young and the survivors have a high risk of acute or late toxicity associated with therapy [7], more efficient and less toxic therapeutic strategies are needed. Targeting signaling pathways offers an attractive approach.

The PI3K/Akt/mTOR pathway is activated in a number of human neoplasms, accompanied by lower overall and disease free survival [8]. This pathway plays a key role in the regulation of cellular functions such as survival, proliferation, cell death and metabolic activities [9]. mTOR (mammalian target of rapamycin) - an important component of this network - is a serine-threonine kinase, which exists in two distinct multiprotein complexes (mTORC1 and mTORC2 - containing characteristic elements: Raptor and Rictor, respectively) [10]. The best known targets of mTORC1 are eukaryotic initiating factor-4E binding proteins (4EBP) and S6 kinase (S6K). mTORC2 can regulate Akt dependent antiapoptotic and survival mechanisms by phosphorylating Akt [11].

The PI3K pathway can be activated by several upstream receptors (IGF-R, Flt3, c-Kit, Notch, TCR, BCR) or intracellular proteins (Ras, BCR/ABL) in various hematological diseases [12]. Information about mTOR activity is very limited; however, transforming direct genetic modifications of PI3K, Akt, mTOR or PTEN are rare - such mutations occur in $5 \%$ of lymphoid malignancies [13]. mTOR has indeed been proven an important element in tumorigenesis in mantle cell lymphoma
(MCL): its role was confirmed in MCL cell proliferation, mainly by influencing cyclin D1 expression [14]. This suggests that the mTOR pathway may play an important role in the development or progression of other lymphoma types as well, and can be considered as a useful therapeutic target.

Rapamycin (and its analogs: rapalogs) interacts with the FKBP12 protein, an element of the mTOR complex, and preferentially disrupts mTORC1 activity [15]. The response of mTORC2 to rapalogs remains conflicting [16]. Rapalogs have been used as immunosuppressive agents in organ transplantation since 1999, and they have been introduced into clinical oncology as a treatement option in renal cell carcinoma and recently in MCL as well [14]. Several trials using mTOR inhibitors in tumors with high mTOR activity are currently underway [17-19].

The aim of our study was to investigate mTOR activity in different lymphomas, with a focus on HL. We found that the majority of HL cases (93\%) displays high mTOR activity. Therefore we suggest that mTOR inhibition (e.g. by rapalogs) may be considered as a therapeutic option in $\mathrm{HL}$, especially in patients with poor prognosis/relapse.

\section{Methods}

\section{Cell culture}

KM-H2, L428, L1236, HDLM2, DEV (Hodgkin-lymphoma) cell lines were cultured in RPMI 1640 supplemented with $100 \mathrm{U} / \mathrm{ml}$ penicillin, $100 \mathrm{ng} / \mathrm{ml}$ streptomycin (Sigma) and heat-inactivated 10\% FCS (Gibco). The UH-01 (HL) cell line was cultured in Iscove's MDM + RPMI-1640 (4:1) supplemented with 20\% FCS, 2 mM L-glutamine (Sigma) and penicillin and streptomycin as above.

Cells were treated with rapamycin (50 ng/ml, Sigma) for $72 \mathrm{~h}$; culture medium was refreshed with new medium supplemented with rapamycin after $72 \mathrm{~h}$ to avoid rapamycin concentration decrease (due to metabolic degradation) in longer treatments (96-144 h). Combination treatments in HL cell lines were done for 72 hours. Doxorubicin (0.2 $\mu \mathrm{M}$; Ebewe Pharma), vincristine (10 nM; Richter Gedeon) and etoposide ( $1 \mu \mathrm{M}$; Pharmachemie BV) were used in combination with rapamycin. Cell morphology was evaluated on methanol fixed and hematoxylineosin (HE) stained cytospin preparates. 


\section{Western-blotting}

Whole cell extracts were prepared and quantitated with Quant-iT protein assay (Invitrogene). Protein extracts $(112.5 \mu \mathrm{g})$ were transferred to PVDF membranes after SDS-PAGE. Membranes were incubated with antiphospho-mTOR (Ser2448), anti-mTOR, anti-phosphop70S6K (Thr389) and anti-phospho-S6 (Ser235/236) antibodies (Cell Signaling), followed by biotinylated secondary antibodies and avidin-HRP complex (Vectastain Elite ABC Kit, Vector), and detected by enhanced chemiluminescence (Pierce ECL Western Blotting Substrate). Membranes were stripped (Re-Blot Plus, Millipore) and reprobed with $\beta$-actin (A2228; Sigma) to confirm equal protein loading.

\section{Enzyme-linked immunosorbent assay (ELISA)}

Cell lysates were obtained from isolated normal B- and T-cells, normal mononuclear cells from buffy coat and lymphoma/leukemia cell lines $\left(5 \times 10^{6}\right.$ cells/sample) in lysis buffer (Cell Signaling) containing $1 \mathrm{mM}$ phenylmethylsulfonyl fluoride (PMSF) for 30 minutes on ice. Sandwich ELISA Kit (p4EBP1 - Thr37/Thr46, Cell Signaling) was used for the detection of phospho-4EBP1 according to the manufacturer's instructions. Optical density (OD) was measured at $450 \mathrm{~nm}$ wavelength.

\section{Flow cytometry}

For apoptosis detection cells were fixed in $70 \%$ ethanol $\left(-20^{\circ} \mathrm{C}\right)$ followed by alkalic extraction $\left(200 \mathrm{mM} \mathrm{Na}_{2} \mathrm{HPO}_{4}\right.$, pH 7.4 and $100 \mathrm{mg} / \mathrm{ml}$ RNase; Sigma) and propidiumiodide staining ( $1 \mathrm{mg} / \mathrm{ml}$, Sigma) according to Mihalik et al. [20]. A minimum of 10,000 events/sample were acquired on a FACScan flow cytometer (BD Biosciences, Erembodegem, Belgium). Data were analyzed with WinList software (Verity Software House, Topsman, ME, USA).

Tissue microarray (TMA) and Hodgkin-lymphoma patients Formalin-fixed paraffin-embedded biopsy specimens from 105 lymphoma patients (6 Burkitt-lymphomas [BL], 23 HL, 11 MCL, 9 anaplastic large-cell lymphomas [ALCL], 9 diffuse large B-cell lymphomas [DLBCL], 12 marginal zone lymphomas [MZL], 13 chronic lymphoid leukemias/ small lymphocytic lymphomas [CLL], 10 follicular lymphomas, 12 peripheral T-cell lymphomas) were included in the first TMA study. The total number of HL patients was 83 in the second TMA set, which represented all HL subtypes: nodular lymphocyte predominant (NLPHL) and classical HL (cHL) types (7 and 76 cases, respectively). cHL samples included nodular sclerosis $(n=47)$, mixed cellularity $(n=18)$, lymphocyte rich $(n=8)$ and lymphocyte depleted $(n=3)$ cases. In each case, two representative cores of $2 \mathrm{~mm}$ diameter were selected from different areas. Reactive lymphoid tissues (tonsils and lymph nodes) were also included as non-neoplastic controls.
Hodgkin-lymphoma patients (40 females, 43 males; age: $8-82$ years [ 23 patients $<18$ years, 41 patients: $18-$ 45 years, 15 patients: $>45$ years]; mean age: 29.8 years) were diagnosed at our Institute between 2000 and 2007. The minimum follow-up period was 5 years in all cases. Clinical data were available in detail in 72 cases from the analyzed 83 patients: 59 of these patients were in complete remission after 5 years of follow-up, 25 patients had relapse and 10 patients died, 13 patients had stem cell transplantation. $60 \%$ of these relapsed patients $(15 / 25)$ are now in $\mathrm{CR}$, including 8 patients who achieved CR following stem cell transplantation. The majority $(64 \%)$ of the patients had stage I-II disease, whereas $36 \%$ presented with stage III-IV disease; $30 \%$ of the patients had B-symptoms.

For pediatric and adolescent patients (8-18 years), treatment group (TG) 1 (stages IA/B, IIA) received 2 cycles OPPA (females) or OEPA (males); TG2 (stages IIB, IIIA, $\mathrm{I}_{\mathrm{E}} \mathrm{A} / \mathrm{B}, \mathrm{II}_{\mathrm{E}} \mathrm{A}$ ) received 2 cycles OPPA or OEPA and 2 cycles COPP; TG3 (IIIB, IVA/B, $\mathrm{II}_{\mathrm{E}} \mathrm{B}, \mathrm{III}_{\mathrm{E}} \mathrm{A} / \mathrm{B}$ ) received 2 cycles OPPA or OEPA and 4 cycles COPP. Additional radiotherapy and/or autologous/allogeneic hematopoietic stem cell transplantation (HSCT) was given in the case of incomplete remission. (OPPA: vincristine, procarbazine, prednisone, doxorubicin; OEPA: vincristine, etoposide, prednisone, doxorubicin; COPP: cyclophosphamid, vincristine, procarbazine, prednisone).

Adult patients were treated with ABVD; DHAP protocol was used in the case of ABVD-resistance. DHAP was also given before HSCT. (ABVD: adriamycin, bleomycin, vinblastine, dacarbazine; DHAP: dexamethasone, high dose cytarabine, cisplatin).

All protocols were approved by the Institutional Ethical Review Board (TUKEB no. 7/2006).

\section{Immunocytochemistry/Immunohistochemistry (ICC/IHC)}

Four $\mu \mathrm{m}$ TMA sections were deparaffinized. Endogenous peroxidase blocking was followed by antigen retrieval in sodium citrate $(\mathrm{pH}=6)$ buffer in a microwave oven.

Cytospin preparates were fixed in $80 \%$ methanol (10,$20^{\circ} \mathrm{C}$ ), and incubated with primary antibodies following endogenous peroxidase blocking.

Slides were incubated overnight at $4^{\circ} \mathrm{C}$ with phospho-S6 (Ser235/236), phospho-mTOR (Ser2448), phospho-4EBP1 (Thr37/46), phospho-p70S6K (Thr389), phospho-HistoneH3 (pHH3), cleaved/activated caspase3 (Cell Signaling), Rictor (Abcam), Raptor (Novus), CD15 (Leica), CD30, MUM-1, Bcl-xL, Bcl-2 (Dako), NF-kappaB-p50 and Survivin (LabVision) antibodies.

Primary antibodies were followed by Novolink Polymer Detection System (Novocastra, Wetzlar, Germany), visualized by DAB and counterstained with hematoxylin. Immunostainings were evaluated by 2 independent pathologists. 3DHistech Pannoramic Viewer program and Nikon E200 were used for tissue microarray analysis. 
Phospho-mTOR, phospho-4EBP1, phospho-p70S6K, phospho-S6 TMA immunostaining reaction intensity (negative, $1+($ weak $) / 2+($ moderate $) / 3+$ (strong) positive) was agreed upon before blind evaluation of the scores $(0 / 1+/ 2+/ 3+)$. Non malignant, reactive lymphocytes showed a maximum positivity of $1+$, whereas plasma cells were score $3+$.

The most reliable phospho-protein marker for mTOR activity was phospho-S6, which is supported by literature data. Therefore, the cases in our study were considered to have high mTOR activity only when scores were $2+/ 3+$ for phospho-S6 and for at least one additional mTOR activity related phosphoprotein (pmTOR, pp70S6K), as described previously [21].

NF-kappaB-p50 was considered positive when nuclear staining was observed; $\mathrm{Bcl}-2$ and $\mathrm{Bcl}-\mathrm{xL}$ positivity was cytoplasmic. Survivin showed both nuclear and cytoplasmic positivity.
The cutoff for positivity was set at $10 \%$ of the tumor cells staining for the antibodies, according to Sebestyén et al. [21].

\section{Hodgkin-lymphoma xenograft model}

Xenograft tumors were established in SCID mice by injecting $2 \times 10^{7} \mathrm{KMH} 2$ cells subcutaneously (s.c.) with matrigel into the back region of 8-10 week old (20-23 g) mice. Palpable tumors were removed, cut into pieces and transplanted into secondary recipient mice. When palpable s.c. tumors developed (after 8 weeks), animals were divided into control and rapamycin-treated groups $(n=10$ each). Rapamycin (Rapamune $1 \mathrm{mg} / \mathrm{ml}$, Wyeth Europa Ltd.) was administered by gavage at $3 \mathrm{mg} / \mathrm{kg}$ body weight three times per week for 8 weeks. Control groups were treated with saline. Body weight and tumor diameter was measured weekly. Tumor volume was calculated as follows: $\Pi / 6 \times(2 \times$ shorter diameter + longer diameter $) / 3)^{3}$.
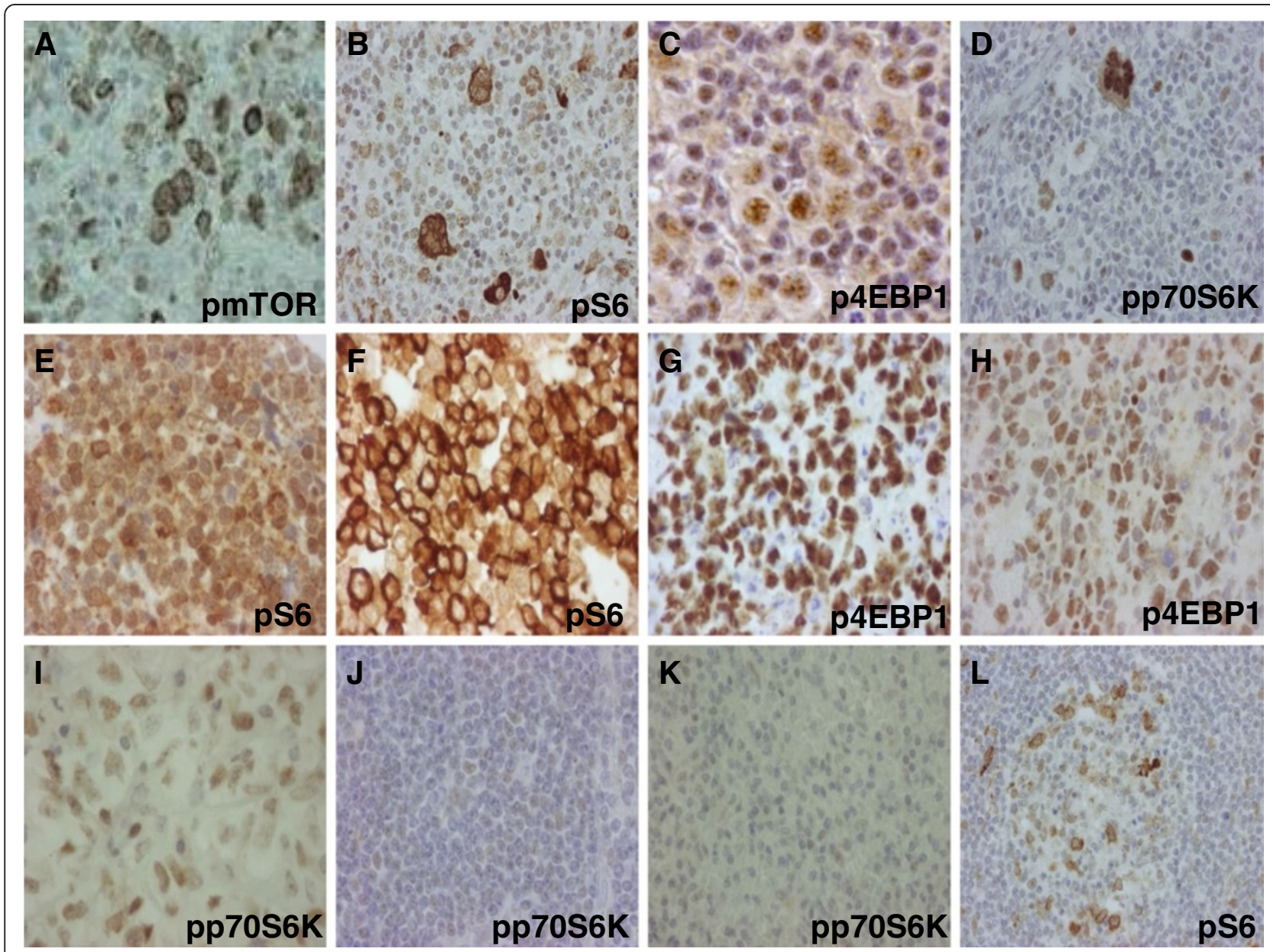

Figure 1 mTOR activity is increased in lymphoma cells. mTOR activity related phosphoproteins in different lymphomas detected by IHC. Lymphomas with high mTOR activity (2+/3+): Hodgkin-lymphoma (A-D), mantle cell lymphoma (E), Burkitt-lymphoma (F-G), diffuse large B-cell lymphoma $(\mathbf{H})$, anaplastic large-cell lymphoma (I). Lymphomas with low mTOR activity $(0 / 1+)$ comparable to non malignant lymphoid cells: chronic lymphoid leukemia/small lymphocytic lymphoma (J), marginal zone lymphoma (K); control lymph node (L); (IHC), 200X, 400X. 


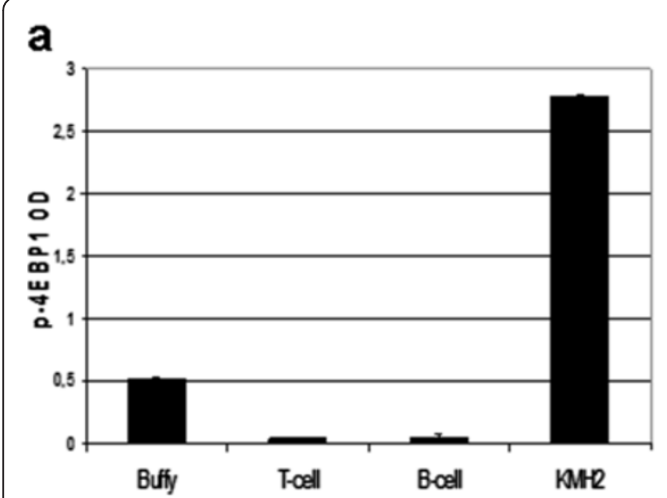

b

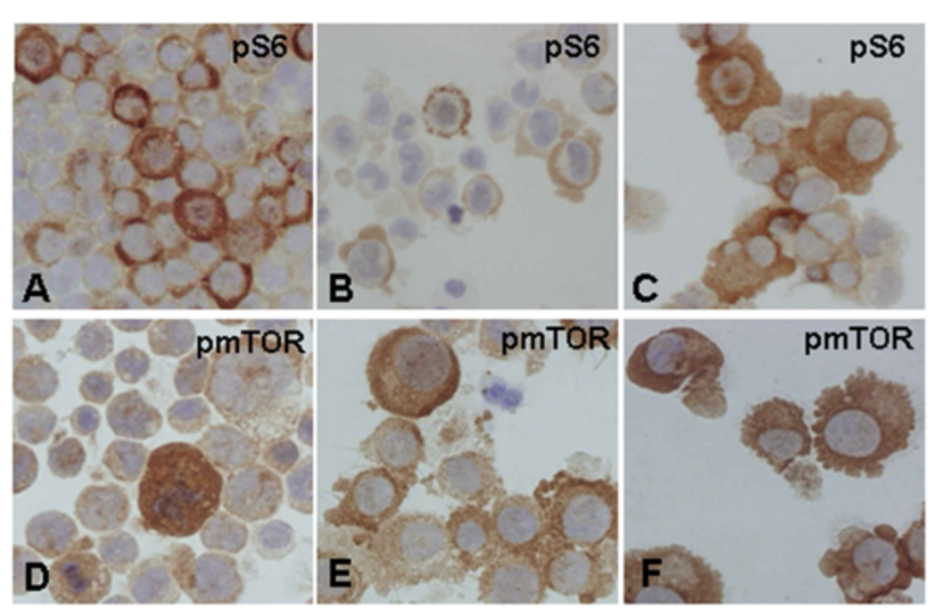

C

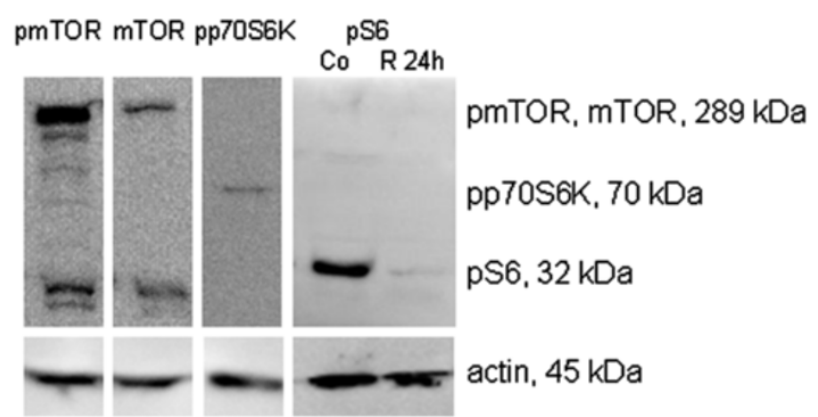

Figure 2 mTOR signaling activity is increased in Hodgkin-lymphoma cell lines. a. The amount of phosphorylated 4EBP1 protein is elevated in $\mathrm{KMH} 2$ Hodgkin-lymphoma cell line compared to normal B-cells, T-cells and buffy coat samples. (ELISA; $p<0.05)$ b. Confirmation of mTOR activity in HL cell lines (KMH2, L428, UH-01, DEV, L1236, HDML2) by pS6 (A-C) and pmTOR (D-F); ICC (400X). c. mTOR kinase and phosphorylated proteins related to its activity (pmTOR, pp70S6K and pS6) in Hodgkin-lymphoma cells detected by Western-blotting. mTOR activity is rapamycinsensitive in $\mathrm{HL}$ cell lines (Co: control; R: rapamycin-treated cells). Representative results showed in $\mathrm{KMH} 2 \mathrm{HL}$ cell line.

Tumor weight was measured in euthanized animals at the end of the experiments. Tumor tissues were formalinfixed, paraffin-embedded and immunostained with human CD15, human CD30, cleaved/activated caspase3 and pHH3. pHH3 and cleaved/activated caspase3 stainings were analyzed with Mirax Viewer software (analysing 4 areas in each sample).

All experiments involving laboratory animals were done in accordance with the Guidelines for Animal Experiments of the Office of Agricultural Administration of Budapest and by the Animal Research Comittee of our university (permission number: 201/2010).

\section{Statistics}

Statistics was calculated with paired Student's t-test, Chi square test and Fisher'exact test using SPSS (SPSS Inc., Chicago, IL, USA) and PAST softwares (PAST free software was downloaded from http://folk.uio.no), and logrank test using GraphPad software (GraphPad, San Diego, California, USA).

\section{Results}

mTOR activity is increased in lymphoma cells

mTOR activity was estimated by immunohistochemistry (IHC) with antibodies against the active form of mTOR and its target proteins on tissue microarray (TMA) sections representing different lymphomas. The evaluation of the mTOR activity stainings of lymphoma subtypes showed high mTOR activity in the majority $(>50 \%)$ of mantle cell lymphoma (11/11), Burkitt-lymphoma (6/6), diffuse large B-cell lymphoma (5/9), anaplastic large-cell lymphoma (8/9) and Hodgkin-lymphoma cases (23/23). Compared to normal lymphoid tissues, HRS cells showed $2+/ 3+$ positivity in virtually all Hodgkin-lymphoma samples in this first TMA study set (containing a limited number of cases). Regarding the analyzed cases of other lymphoma types, no or only low $(0 /+)$ mTOR activity was detected in marginal zone lymphomas, chronic lymphoid leukemias/small lymphocytic lymphomas and peripheral T-cell lymphomas (8/12, 12/13 and 10/12 negative/low, respectively; Figure 1). IHC results were conflicting in follicular lymphoma cases, because 7/10 samples were 


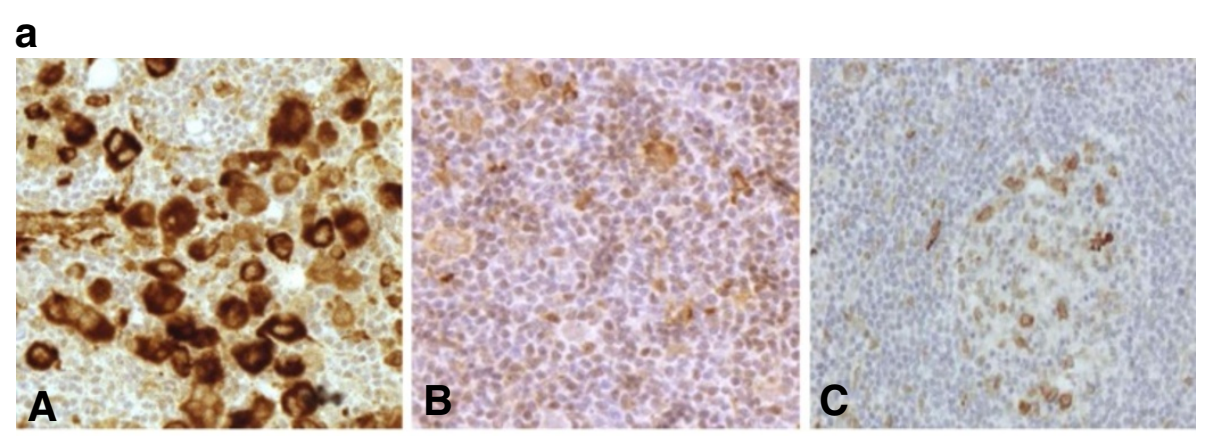

b

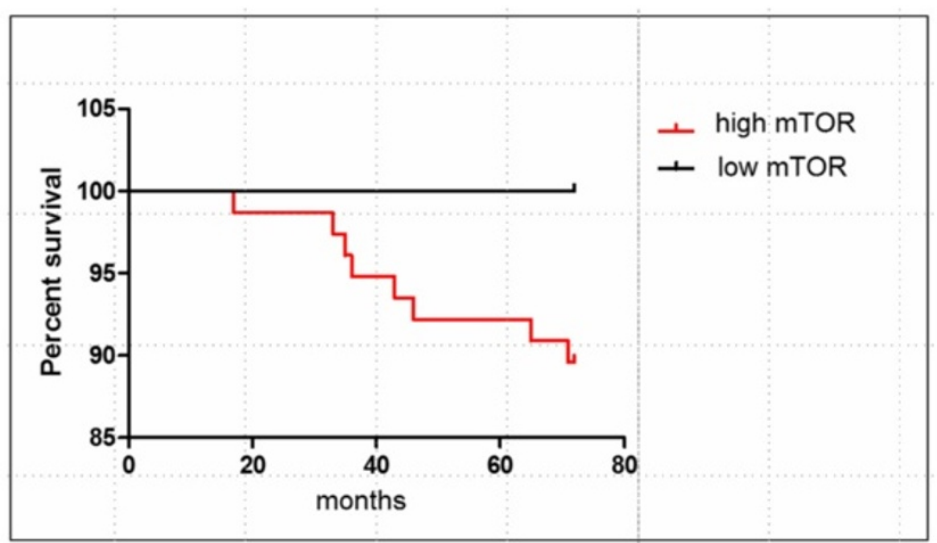

Figure 3 mTOR activity in human Hodgkin-lymphoma biopsies. a. High (A) and low (B) mTOR activity in lymphoma cells of Hodgkinlymphoma biopsies. Low mTOR activity is comparable to that of reactive lymph nodes (C). (pS6 IHC; representative examples are shown.) b. Kaplan-Meier survival curves stratified by mTOR activity: low mTOR and high mTOR groups include 6 and 77 cases respectively ( $p=0.42$ ).

positive for pmTOR, and 6/10 were positive for pp70S6K, but all samples were negative for pS6.

\section{Hodgkin-lymphoma is characterized by high mTOR activity}

HL cell lines - KMH2, UH-01, L428, L1236, HDLM2 and DEV - showed high mTOR activity by ICC (Figure $2 \mathrm{~b}$ ). ICC results were confirmed by both Western-blotting and ELISA in KMH2 cells, and either Western-blotting or ELISA was performed in the other cell lines as well (Figure 2).

A second set of TMA was constructed containing biopsy specimens from $83 \mathrm{HL}$ patients. High mTOR activity was confirmed as a characteristic feature of HL (77/83), independently from the subtypes (NS: 44/47, MC: $17 / 18$, LR: 8/8, LD: 3/3, NLPHL: 5/7) (Figure 3a). Non-malignant lymphoid tissues (tumor infiltrating lymphocytes, reactive tonsils and lymph nodes) showed low expression (0/1+) of mTOR-related phospho-proteins. IHC results were compared to the clinical data from 72 patients with long-term (a minimum of five-year) follow-up; we did not find a significant correlation with age, gender, stage, prognosis and histopathological type. We observed a tendency of correlation with therapeutic response and the present status of patients, but it did not reach statistical significance $(\mathrm{p}=0.42)$ (Figure $3 \mathrm{~b})$. It should be mentioned that all cases with low mTOR activity (6/72) were in complete remission with at least 5-year disease-free survival. Moreover, high mTOR activity $(2+/ 3+)$ was detected in the biopsies of all patients who had poor prognosis and died (11/72). However, high mTOR activity was observed in the case of both favorable and unfavorable clinical response.

We found that the expression of Raptor and Rictor (characteristic proteins of mTORC1 and mTORC2, respectively) by IHC was similar to the expression pattern of normal lymphocytes in $82 \mathrm{HL}$ cases (Figure $4 \mathrm{a}$ ). Rictor overexpression $(2+/ 3+)$ (which was detected in several control breast carcinomas, indicating potential mTORC2 dominant expression) was detected only in one HL case.

Anti-apoptotic proteins (Bcl-2, Bcl-xL, Survivin and NF-kappaB-p50) known to be overexpressed in HLs were analyzed to search for a potential correlation and the role of mTOR activity behind their expression in HL (Figure 4b). High Bcl-xL expression was seen in the cytoplasm of HRS cells in all cases. NF-kappaB-p50 was expressed in $70 \%$ of HRS cells. $30 \%$ and $65 \%$ of the analyzed HL cases showed Bcl-2 and Survivin expression, 


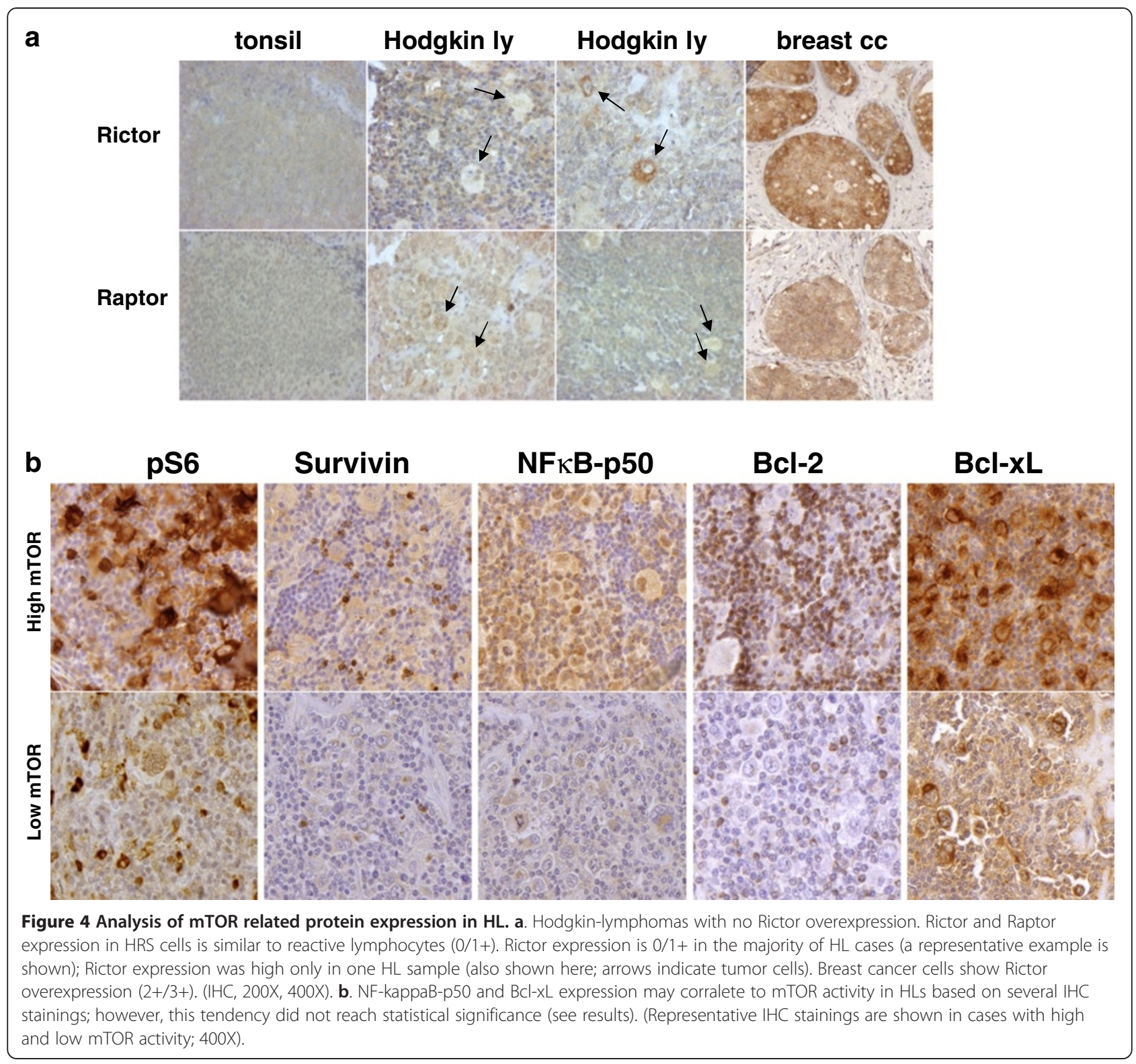

respectively, which was significantly lower than the number of mTOR active cases. Based on these results, Bcl-xL and NF-kappaB-p50 expression may correlate with mTOR activity in HLs, but we did not find significance with Fisher's exact test ( $\mathrm{p}=0.07$ and $\mathrm{p}=0.86$, respectively); however, statistical analysis was hampered by the low number of cases with low mTOR activity.

\section{mTOR activity can be targeted in HL cells, leading to} growth inhibition in vitro and in vivo

Rapamycin treatment lead to G1 cell cycle block in all HL lymphoma cell lines without apoptosis induction after $72 \mathrm{~h}$ (Figure 5a). However, a longer (96-144 h) in vitro rapamycin treatment was able to switch on the apoptotic program (Figure 5b). The level of phosphorylated S6 was remarkably decreased, further supporting the inhibition of mTOR activity in HL cell lines (Figure 2c).

We investigated the effect of rapamycin combined with chemotherapeutic agents in KMH2, DEV and L1236 HL cell lines. When given in combination, rapamycin significantly increased the apoptotic effect of low dose "traditional" chemotherapeutic agents (doxorubicin, vincristine and etoposide) in $\mathrm{KMH} 2$ and DEV cell lines (Figure 5c). Rapamycin treatment had only an antiproliferative effect in L1236 cells, and could not enhance apoptosis induced by chemotherapeutic agents.

The in vivo growth inhibitory effect of rapamycin was also confirmed in SCID mice with KMH2 Hodgkinlymphoma xenografts. Rapamycin treatment (8 weeks) significantly reduced tumor volume and tumor weight in 

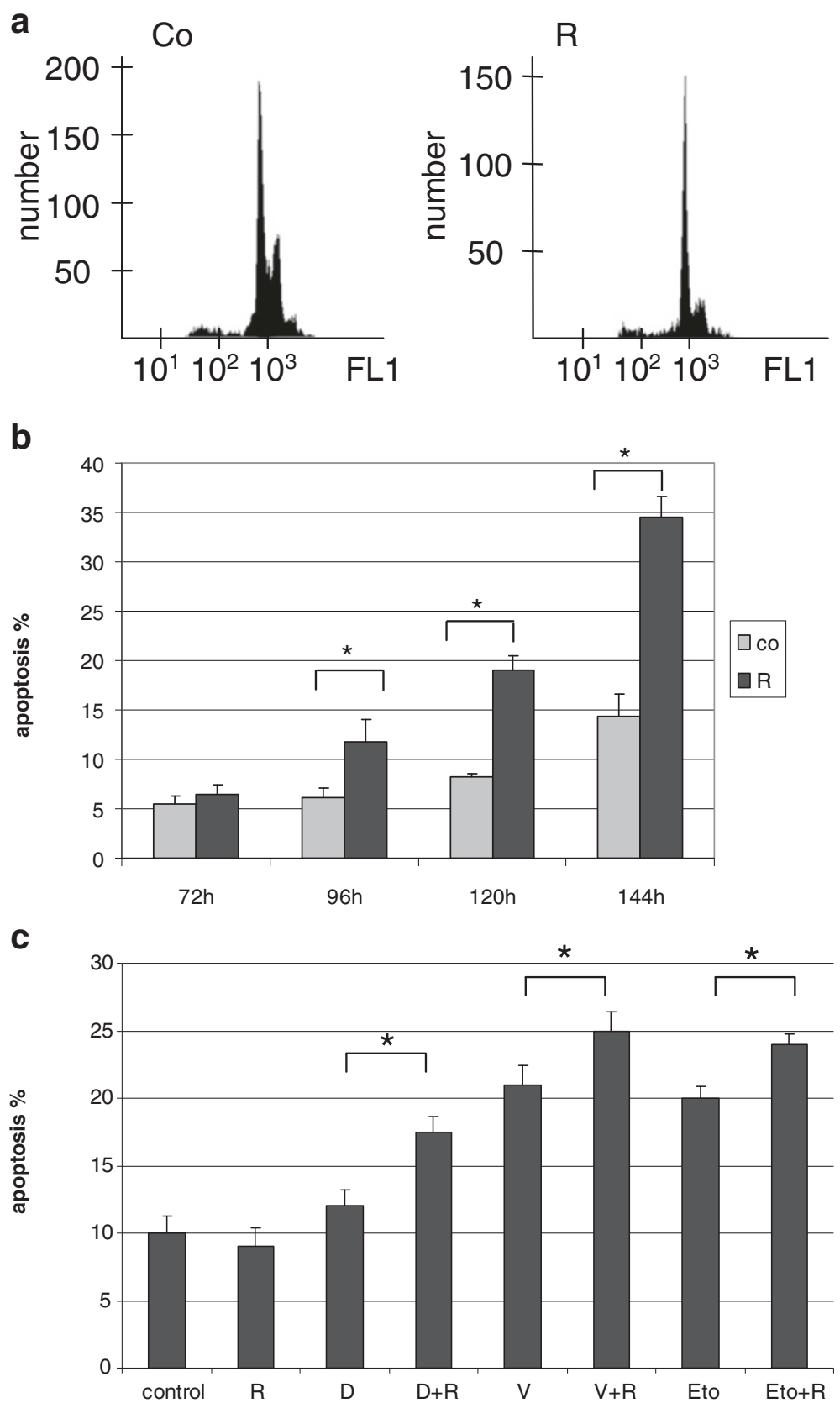

Figure 5 Anti-proliferative and apoptotic effects of rapamycin in vitro. a. G1 cell cycle arrest detected by flow cytometry after $72 \mathrm{~h}$ rapamycin treatment $(50 \mathrm{ng} / \mathrm{ml})$ in $\mathrm{KMH} 2$ Hodgkin-lymphoma cells. b. The apoptotic effect of rapamycin is time dependent in $\mathrm{KMH} 2 \mathrm{cells}$ (flow cytometry, ${ }^{*}:$ < 0.05$)$. c. Rapamycin treatment increased the apoptotic effect of chemotherapeutic agents in HL cell lines after 24 h. A representative experiment in $\mathrm{KMH} 2$ cells is shown here. (R: rapamycin $50 \mathrm{ng} / \mathrm{ml}$, D: doxorubicin $0.2 \mu \mathrm{M}$, V: vincristine 10 nM, Eto: etoposide 1 $\left.\mu \mathrm{M} ;{ }^{*}: \mathrm{p}<0.05\right)$.

the treated animals (Figure 6a). The average tumor weight was $0.65 \mathrm{~g}$ vs. $0.25 \mathrm{~g}$ in the control vs. treated group, respectively. The significant anti-proliferative and apoptotic effect of in vivo treatment was also confirmed in KMH2 xenograft biopsies: the number of phosphoHistone $\mathrm{H} 3$ ( $\mathrm{pHH} 3$; mitotic marker) positive cells were decreased (30\% compared to control) and the number of cleaved/activated caspase3 (apoptotic marker) positive cells were increased $(7.3 \times$ compared to control) in treated tumors (Figure 6b).

\section{Discussion}

The introduction of new drugs has to be based on convincing evidence in malignancies where clinical response 

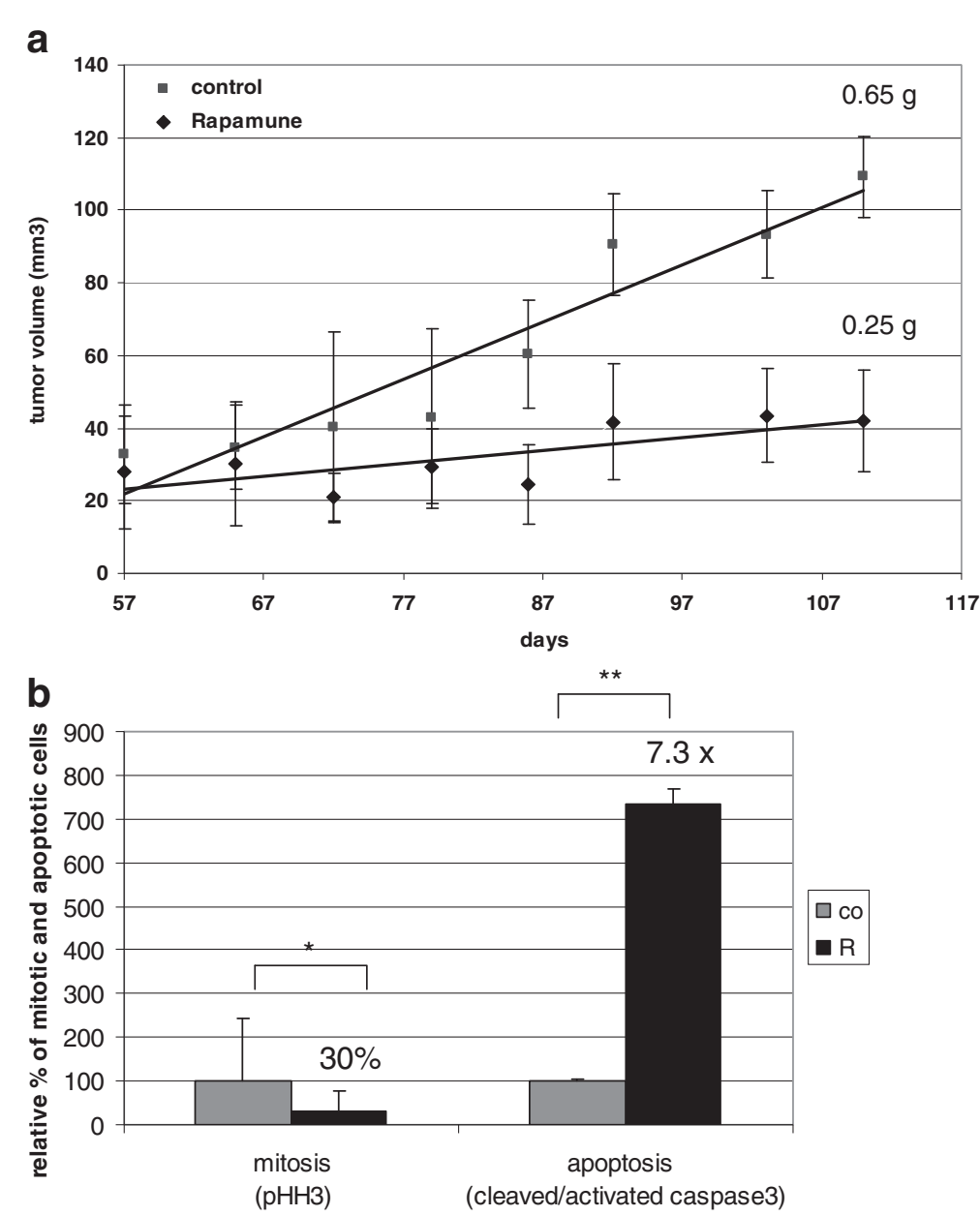

Figure 6 Rapamycin inhibits tumor growth in vivo. a. Rapamycin inhibits tumor growth in KMH2 (Hodgkin-lymphoma) xenografts in vivo. Tumor volumes are shown in control and rapamycin-treated mice during an 8-week treatment. Tumor weight at the end of the experiment is also indicated; tumors in rapamune treated mice were significantly smaller $(p<0.05)$. b. Confirmation of the in vivo anti-proliferative and apoptotic effect of rapamycin by IHC detection of $\mathrm{pHH} 3$ (proliferation marker) and cleaved/activated caspase3 (apoptosis marker) $\left(^{*}: p<0.05,{ }^{* *}: p<0.01\right)$.

rate (or even cure rate) is rather high. A typical example is Hodgkin-lymphoma (HL); in fact, no new drugs have been approved by the FDA for HL in the last 30 years [22]. However, treatment failures in patients with advanced disease, insufficient response (recurrences and resistance) as well as late toxicity of the currently used chemotherapy - including second malignancies, cardiovascular toxicity and infertility - requires improvement in standard options for treating HL [23]. Targeted therapy is an innovative research field in oncology, where the defects of major regulatory steps fine-tuning critical cell functions such as survival, proliferation and apoptosis serve as molecular targets.

There is substantial evidence highlighting the importance of changes in the activity of different PI3K pathway members, including mTOR complexes. Here we show that mTOR activity is a characteristic feature in the majority ( $>50 \%$ ) of MCL, BL, DLBCL, ALCL and HL cases.
High mTOR activity of HRS cells is further supported by our second TMA study focusing on HLs.

Previous publications reported only small numbers of cases without considering subclassification of HL $[24,25]$. Based on the evaluation of different downstream mTOR target proteins in $83 \mathrm{HL}$ cases, increased mTOR activity was confirmed in more than $90 \%$ of HLs in our work, which was independent of HL subtype and clinical parameters. Low mTOR activity cases had no relapse, and these patients had more than 5 year disease free survival, with complete remission. However, high mTOR activity was observed in the case of both favorable and unfavorable clinical response, therefore it cannot be considered as a prognostic indicator. We are aware that the 83 HL patients included in our study comprise a heterogeneous patient group in respect of age, gender, stage, histological type and prognosis. Therefore, it is difficult to reach significant conclusions; nevertheless, our study 
offers a comprehensive overview of this heterogeneous group, which is obviously characterized by high mTOR activity in general.

At a molecular level, mTOR activity is known to play a role in cyclin D1 overexpression and cell cycle dysregulation in MCL [14]. Through the regulation of translation or by directly influencing the activity of p70S6K, mTOR can induce the antiapoptotic functions of mitochondrial proteins, e.g. by BAD phosphorylation, supporting the survival and proliferation of tumor cells [26]. The malfunction of apoptotic pathways and the overexpression of several cyclins (cyclin A, B1 and E) are also known in HL [27]. The overexpression of antiapoptotic signals (Bcl-xL) showed correlation with high mTOR activity in our study.

Each time a protein known to be a member of regulatory signaling pathways, participating in the development and/or progression of malignancies is brought into focus, the question arises: can we turn our knowledge to therapeutic advantage? In the case of mTOR, inhibitors already exist (rapamycin and its analogs: rapalogs), which are well tolerated [28], and rapamycin has also been shown to synergize with anticancer agents in several tumors [12,29-31]. Rapalogs/rapamycin inhibited proliferation and induced apoptosis, moreover, they increased the apoptotic effect of chemotherapeutic agents (doxorubicin, vincristine and etoposide) in HL cells in our xenograft and in vitro experiments. These results - along with others [32-35] - suggest that mTOR inhibition is an option in tumors with increased mTOR activity. In this respect $\mathrm{HL}$ could be a good candidate, as high $\mathrm{mTOR}$ activity and mTORC1 expression could be detected in a high percentage of cases, and mTORC1 inhibition also had an antiproliferative and apoptotic effect in vitro and in vivo.

The efficiency of mTOR inhibitors may be dependent on the ratio of mTOR complexes [36]. While mTORC1 is sensitive to currently used mTOR inhibitors, the rapalog sensitivity of mTORC2 is still conflicting, and may vary in different cell types [37,38]. New dual inhibitors inhibiting both mTOR complexes, or mTORC1 and upstream elements of the PI3K/Akt/mTOR pathway - are being developed [39]. The inclusion of upstream proteins is quite logical, because the inhibition of mTORC1 may be able to activate them. The immunohistochemical detection of the phosphorylated forms of Akt (specifically, Ser473, which is connected to mTORC2) is very difficult. We tested different antibodies but we could not detect realiably specific staining in our lymphoid tissues. Baker et al. investigated the stability of phosphorylated Akt and they established that postoperative surgical samples may be of limited value for measuring phospho-Akt levels because Akt can be dephosphorylated quickly during tumor removal and fixation [40]. Considering this, we chose to investigate the expression of Rictor, one essential component of functioning mTORC2. We concluded that mTORC2 was not a characteristic feature when Rictor expression was not detected in the samples. Several solid and lymphoid malignancies such as non-GC DLBCLs overexpress Rictor (a characteristic protein in $\mathrm{mTORC} 2$ ), which potentially indicates increased mTORC2 activity $[21,41,42]$. Rictor was not overexpressed in our HL cell lines and cases, which can explain the sensitivity to rapamycin/rapalogs.

Taken together, Hodgkin-lymphoma is characterized by high mTOR activity, and this high mTOR activity does not exclude good prognosis. Moreover, mTORC1 may be a potential therapeutic target in HL, especially when commonly used protocols prove ineffective, and may also allow dose reduction of chemotherapeutic drugs in order to decrease late toxicity without diminishing treatment efficacy. The combination of mTOR inhibitors with other agents targeting critical molecular sites will likely be crucial for achieving the best clinical response.

\section{Conclusion}

Based on our results, mTOR activity may be a potential therapeutic tool in different lymphoma types. In particular, the majority of Hodgkin-lymphomas have high mTOR activity (with no mTORC2/Rictor expression). These data, along with our in vitro and in vivo results with mTOR inhibitors suggest that the inhibition of mTORC1 may be feasible in the therapy, especially in Hodgkin-lymphomas when standard protocols prove ineffective. The combination of mTOR inhibitors with other agents will probably offer the highest efficiency for achieving the best clinical response, and may also allow dose reduction in order to decrease late treatment toxicity in these cases.

\section{Abbreviations}

ALCL: Anaplastic large-cell lymphoma; BL: Burkitt-lymphoma; CHL: Classical Hodgkin-lymphoma; CLL: Chronic lymphoid leukemia/small lymphocytic lymphoma; CR: Complete remission; DLBCL: Diffuse large B-cell lymphoma; HE: Hematoxylin-eosin; HL: Hodgkin-lymphoma; HRS cell: Hodgkin-/ReedSternberg cell; HSCT: Hematopoietic stem cell transplantation;

LD: Lymphocyte depleted; LR: Lymphocyte rich; MC: Mixed cellularity; MCL: Mantle cell lymphoma; mTOR: Mammalian target of rapamycin; mTORC: mTOR complex; NLPHL: Nodular lymphocyte predominant; NS: Nodular sclerosis; pHH3: phospho-Histone H3; PI3K: Phosphatidylinositol3-kinase; pmTOR: phoshpo-mTOR; p70S6K: p70S6 kinase; pp70S6K: Phosphop70S6 kinase; pS6: Phospho-S6; p4EBP1: phospho-4EBP1; SCID: Severe combined immunodeficiency; S6K: S6 kinase; TG: Treatment group; TMA: Tissue microarray; 4EBP1: Eukaryotic translation initiating factor 4E-binding protein1.

\section{Competing interests}

The authors declare that they have no competing interests.

\section{Authors' contributions}

AS was the principal investigator, designed the study, supervised materials, data collection and analysis, and takes primary responsibility for the paper. ÁM, NN and TBS designed and prepared TMA blocks and performed IHC stainings and statistical analysis for this study. MH, LB, LK, JCs took part in the morphological evaluation of sections and evaluated IHC results. ÁM, NN and 
W performed in vitro and in vivo experiments, MCs and ZsV collected clinical data for the study and participated in analysis. AS, MH, ÁM and KL wrote the paper. All authors read and approved the final manuscript.

\section{Acknowledgements}

The authors would like to thank Tibor Krenács, Renáta Kis, Edit Parsch, Zsuzsa Kaminszky, András Sztodola and Anna Tamási (1st Department of Pathology and Experimental Cancer Research, Semmelweis University) for technical assistance, and the assistance of hemato-oncologists who treated and followed the patients. This work was supported by OTKA projects (K81624 K68341, K84262, K76204) of the Hungarian Academy of Sciences.

\section{Author details}

1 1st Department of Pathology and Experimental Cancer Research Semmelweis University, Üllői út 26, Budapest 1085, Hungary. ${ }^{2} 2$ nd Department of Pediatrics, Semmelweis University, Tüzoltó u. 7-9, Budapest 1094, Hungary. ${ }^{3}$ Tumor Progression Research Group of Joint Research Organization of the Hungarian Academy of Sciences and Semmelweis University, Budapest, Hungary.

Received: 11 October 2012 Accepted: 25 April 2013

Published: 22 May 2013

\section{References}

1. Marcos-Gragera R, Allemani C, Tereanu C, De Angelis R, Capocaccia R, Maynadie M, Luminari S, Ferretti S, Johannesen TB, Sankila R, KarjalainenLindsberg ML, Simonetti A, Martos MC, Raphaël M, Giraldo P, Sant M, HAEMACARE Working Group: Survival of European patients diagnosed with lymphoid neoplasms in 2000-2002: results of the HAEMACARE project. Haematologica 2011, 96:720-8.

2. Steidl C, Connors JM, Gascoyne RD: Molecular Pathogenesis of Hodgkin's Lymphoma: Increasing Evidence of the Importance of the Microenvironment. JCO 2011, 29:1812-1826.

3. Thomas RK, Re D, Wolf J, Diehl V: Part I: Hodgkin's lymphoma-molecular biology of Hodgkin and Reed-Sternberg cells. Lancet Oncol 2004, 5:11-8.

4. Küppers R, Hansmann ML: The Hodgkin and Reed/Sternberg cell. Int J Biochem Cell Biol 2005, 37:511-7.

5. Favier O, Heutte N, Stamatoullas-Bastard A, Carde P, Van't Veer MB, Aleman BM, Noordijk EM, Thomas J, Fermé C, Henry-Amar M, European Organization for Research and Treatment of Cancer (EORTC) Lymphoma Group and the Groupe d'Etudes des Lymphomes de l'Adulte (GELA): Survival after Hodgkin lymphoma. Cancer 2009, 115:1680-91.

6. Re D, Thomas RK, Behringer K, Diehl V: From Hodgkin disease to Hodgkin lymphoma: biologic insights and therapeutic potential. Blood 2005, 105:4553-60.

7. Currin ES, Gopal AK: Treatment strategies for Hodgkin lymphoma recurring following autologous hematopoietic stem cell transplantation. Korean J Hematol 2012, 47:8-16.

8. Ferté C, André F, Soria JC: Molecular circuits of solid tumors: prognostic and predictive tools for bedside use. Nat Rev Clin Oncol 2010, 7:367-80

9. Laplante M, Sabatini DM: mTOR Signaling in Growth Control and Disease. Cell 2012, 149:274-93.

10. Guertin DA, Sabatini DM: Defining the role of mTOR in cancer. Cancer Cell 2007, 12:9-22

11. Pópulo H, Lopes JM, Soares P: The mTOR Signalling Pathway in Human Cancer. Int J Mol Sci 2012, 13:1886-918.

12. Vu C, Fruman DA: Target of rapamycin signaling in leukemia and lymphoma. Clin Cancer Res 2010, 16:5374-80.

13. Chalhoub N, Baker SJ: PTEN and the PI3-kinase pathway in cancer. Annu Rev Pathol 2009, 4:127-50.

14. Kelly KR, Rowe JH, Padmanabhan S, Nawrocki ST, Carew JS: Mammalian target of rapamycin as a target in hematological malignancies. Target Oncol 2011, 6:53-61.

15. Chapuis N, Tamburini J, Green AS, Willems L, Bardet V, Park S, Lacombe C, Mayeux P, Bouscary D: Perspectives on inhibiting mTOR as a future treatment strategy for hematological malignancies. Leukemia 2010, 24:1686-99.

16. Sabatini DM: mTOR and cancer: insights into a complex relationship. Nat Rev Cancer 2006, 6:729-34.

17. Alvarado Y, Mita MM, Vemulapalli S, Mahalingam D: Clinical activity of mTORIs in solid tumors. Targ Oncol 2011, 6:69-94.
18. Schatz JH: Targeting the PI3K/AKT/mTOR pathway in non-Hodgkin's lymphoma: results, biology, and development strategies. Curr Oncol Rep 2011, 13:398-406.

19. Sheppard K, Kinross KM, Solomon B, Pearson RB, Phillips WA: Targeting PI3 kinase/AKT/mTOR signaling in cancer. Crit Rev Oncog 2012, 17:69-95.

20. Mihalik R, Uher F, Pocsik ÉE, Berczi L, Benczur M, Kopper L: Detection of Drug-induced Apoptosis by Flow Cytometry after Alkaline Extraction of Ethanol Fixed Cells. Pathol Oncol Res 1996, 2:78-83.

21. Sebestyén A, Sticz TB, Márk Á, Hajdu M, Timár B, Nemes K, Nagy N, Váradi Z, Kopper L: Activity and complexes of mTOR in diffuse large B-cell lymphomas - a tissue microarray study. Mod Pathol 2012, 25:1623-8.

22. Jona A, Younes A: Novel treatment strategies for patients with relapsed classical Hodgkin lymphoma. Blood Rev 2010, 24:233-8.

23. DiehI V, Thomas RK, Re D: Part II: Hodgkin's lymphoma-diagnosis and treatment Lancet Oncol 2004, 5:19-26.

24. Dutton A, Reynolds GM, Dawson CW, Young LS, Murray PG: Constitutive activation of phosphatidyl-inositide 3 kinase contributes to the survival of Hodgkin's lymphoma cells through a mechanism involving Akt kinase and mTOR. J Pathol 2005, 205:498-506.

25. De J, Brown RE: Tissue-microarray based immunohistochemical analysis of survival pathways in nodular sclerosing classical Hodgkin lymphoma as compared with Non-Hodgkin's lymphoma. Int J Clin Exp Med 2010, 3:55-68.

26. Vignot S, Faivre S, Aguirre D, Raymond E: mTOR-targeted therapy of cancer with rapamycin derivatives. Ann Oncol 2005, 16:525-37.

27. García JF, Camacho Fl, Morente M, Fraga M, Montalbán C, Alvaro T, Bellas C, Castaño A, Díez A, Flores T, Martin C, Martinez MA, Mazorra F, Menárguez J, Mestre MJ, Mollejo M, Sáez Al, Sánchez L, Piris MA, Spanish Hodgkin Lymphoma Study Group: Hodgkin and Reed-Sternberg cells harbor alterations in the major tumor suppressor pathways and cell-cycle checkpoints: analyses using tissue microarrays. Blood 2003, 101:681-9.

28. Pulsipher MA, Wall DA, Grimley M, Goyal RK, Boucher KM, Hankins P, Grupp $\mathrm{SA}$, Bunin N: A phase $1 /$ II study of the safety and efficacy of the addition of sirolimus to tacrolimus/methotrexate graft versus host disease prophylaxis after allogeneic haematopoietic cell transplantation in paediatric acute lymphoblastic leukaemia (ALL). Br J Haematol 2009, 147:691-9.

29. Seront E, Rottey S, Sautois B, Kerger J, D'Hondt LA, Verschaeve V, Canon JL, Dopchie C, Vandenbulcke JM, Whenham N, Goeminne JC, Clausse M, Verhoeven D, Glorieux P, Branders S, Dupont P, Schoonjans J, Feron O, Machiels JP: Phase II study of everolimus in patients with locally advanced or metastatic transitional cell carcinoma of the urothelial tract: clinical activity, molecular response, and biomarkers. Ann Oncol 2012, 23:2663-70

30. Villarreal-Garza C, Cortes J, Andre F, Verma S: mTOR inhibitors in the management of hormone receptor-positive breast cancer: the latest evidence and future directions. Ann Oncol 2012, 23:2526-35.

31. Li SH, Huang EY, Lu HI, Huang WT, Yen CC, Huang WC, Chen CH: Phosphorylated mammalian target of rapamycin expression is associated with the response to chemoradiotherapy in patients with esophageal squamous cell carcinoma. J Thorac Cardiovasc Surg 2012, 144:1352-9.

32. Jundt F, Raetzel N, Müller C, Calkhoven CF, Kley K, Mathas S, Lietz A, Leutz A, Dörken B: A rapamycin derivative (everolimus) controls proliferation through down-regulation of truncated CCAAT enhancer binding protein beta and NF-\{kappa\}B activity in Hodgkin and anaplastic large cell lymphomas. Blood 2005, 106:1801-7.

33. Jiang $B H$, Liu $L Z$ : Role of $m T O R$ in anticancer drug resistance: perspectives for improved drug treatment. Drug Resist Updat 2008, 11:63-76.

34. Johnston PB, Inwards DJ, Colgan JP, Laplant BR, Kabat BF, Habermann TM, Micallef IN, Porrata LF, Ansell SM, Reeder CB, Roy V, Witzig TE: A Phase II trial of the oral mTOR inhibitor everolimus in relapsed Hodgkin lymphoma. Am J Hematol 2010, 85:320-4.

35. Barnett CM: Everolimus: targeted therapy on the horizon for the treatment of breast cancer. Pharmacotherapy 2012, 32:383-96.

36. Oh WJ, Jacinto E: mTOR complex 2 signaling and functions. Cell Cycle 2011, 10:2305-16.

37. Akcakanat A, Singh G, Hung MC, Meric-Bernstam F: Rapamycin regulates the phosphorylation of rictor. Biochem Biophys Res Commun 2007 , $362 \cdot 330-3$ 
38. Sarbassov DD, Ali SM, Sengupta S, Sheen JH, Hsu PP, Bagley AF, Markhard AL, Sabatini DM: Prolonged rapamycin treatment inhibits mTORC2 assembly and Akt/PKB. Mol Cell 2006, 22:159-68.

39. Khokhar NZ, Altman JK, Platanias LC: Emerging roles for mammalian target of rapamycin inhibitors in the treatment of solid tumors and hematological malignancies. Curr Opin Oncol 2011, 23:578-86.

40. Baker FA, Tomislav D, Nathan TI, Williams R, Fenoglio-Preiser C, Powie G: Stability of Phosphoprotein as a Biological Marker of Tumor Signaling. Clin Cancer Res 2005, 11:4338-4340.

41. Masri J, Bernath A, Martin J, Jo OD, Vartanian R, Funk A, Gera J: mTORC2 activity is elevated in gliomas and promotes growth and cell motility via overexpression of rictor. Cancer Res 2007, 67:11712-20.

42. Gulhati P, Cai Q, Li J, Liu J, Rychahou PG, Qiu S, Lee EY, Silva SR, Bowen KA, Gao T, Evers BM: Targeted inhibition of mammalian target of rapamycin signaling inhibits tumorigenesis of colorectal cancer. Clin Cancer Res 2009, 15:7207-16

doi:10.1186/1471-2407-13-250

Cite this article as: Márk et al:: Characteristic mTOR activity in

Hodgkin-lymphomas offers a potential therapeutic target in high risk

disease - a combined tissue microarray, in vitro and in vivo study. BMC

Cancer 2013 13:250.

\section{Submit your next manuscript to BioMed Central and take full advantage of:}

- Convenient online submission

- Thorough peer review

- No space constraints or color figure charges

- Immediate publication on acceptance

- Inclusion in PubMed, CAS, Scopus and Google Scholar

- Research which is freely available for redistribution 